\title{
Determination of Hydrocarbon-bearing Property of Deep-water Sandstone by Fluid Pressure Gradient from Seismic Attributes
}

\author{
Chang Deshuang, Chen Zhigang*, Han Yuchun, Sun Xing, Chen Jie, Zhao Qian, Zhao Xiaoqing \\ Geological Research Center, Bureau of Geophysical Prospect, Zhuozhou, China
}

Email address:

chenzhigang@cnpc.com.cn (Chen Zhigang)

${ }^{*}$ Corresponding author

To cite this article:

Chang Deshuang, Chen Zhigang, Han Yuchun, Sun Xing, Chen Jie, Zhao Qian, Zhao Xiaoqing. Determination of Hydrocarbon-bearing Property of Deep-water Sandstone by Fluid Pressure Gradient from Seismic Attributes. International Journal of Oil, Gas and Coal Engineering. Vol. 9, No. 3, 2021, pp. 36-41. doi: 10.11648/j.ogce.20210903.11

Received: April 27, 2021; Accepted: May 10, 2021; Published: July 22, 2021

\begin{abstract}
In the same hydrodynamic system, because the densities of oil and gas are smaller than that of water, their pressure gradients are also smaller. Therefore, the pressure gradient can determine fluid properties. Based on seismic data and well logging data, this paper attempts to apply the equivalent medium theory to predict the pressure gradient, and then to identify fluids. Firstly, the upper and lower limits of bulk modulus and shear modulus of rocks can be obtained by using wellbore and well logging interpretation data. Secondly, based on the equivalent medium theory, the fluid velocity (when rock rigidity approaching zero) and the rock matrix velocity (when porosity approaching zero) are predicted. Thirdly, the predicted two types of velocity curves and the original acoustic curves are used for seismic inversion. Finally, according to the inversion results, the formation pressure and pressure gradient can be obtained by using the Fillippone pressure formula, and the hydrocarbon-bearing property of reservoirs can be determined according to the theoretical pressure gradients of different fluids. For offshore deep-water sandstone in an overseas block, when the frequency attributes for hydrocarbon detection cannot reflect hydrocarbons well, the fluid pressure gradient attribute is used to predict hydrocarbons, and the prediction coincidence rate reaches $70 \%$.
\end{abstract}

Keywords: Pressure Gradient, Bulk Modulus, Shear Modulus, Hydrocarbon Detection, Equivalent Medium Theory

\section{Introduction}

In recent years, hydrocarbon detection technologies play increasingly important roles in oil and gas exploration and development. At present, seismic based hydrocarbon detection technologies are classified into two catalogues, pre-stack hydrocarbon detection and post-stack hydrocarbon detection. Pre-stack hydrocarbon detection technologies are mainly AVO related technology which describes the amplitude variation with offset in trace gather, including AVO pre-stack inversion, AVO statistical analysis, AVO anisotropy analysis and AVO pore fluid identification, etc. [1-5] In addition to conventional seismic attributes, post-stack hydrocarbon detection technologies are mainly based on the fact that the existence of hydrocarbon will lead to amplitude variation in different frequency bands. Currently, the widely applied hydrocarbon detection technologies are mainly based on low frequency [6-8] and "low frequency resonance, high frequency attenuation" [9-12]. The pre-stack AVO hydrocarbon detection technologies are more sensitive to natural gas, but they are subject to the quality of pre-stack gathers, thus they need the full combination of processing and interpretation of seismic data to obtain better results, so the workload is huge. What's more, post-stack hydrocarbon detection technologies based on amplitude changes in different frequency bands are largely limited by seismic processing, as many links in processing will lead to changes in spectral characteristics.

In the 1970s-1980s, Fillippone [13] put forward the Fillippone Formula to predict pressure by using the velocity through the research in the Gulf of Mexico. Later, some geophysicists improved the Fillippone Formula and proposed 
Martinez Method [14], Liu Zhen Method [15], and Yun Meihou Method [16] etc. Based on the above methods, seismic prospecting researchers are able to use acoustic logging curve and seismic data to carry out seismic inversion to obtain interval velocity, and then predict formation pressure. However, the defect of these methods is the low accuracy of interval velocity obtained by seismic inversion due to the limitation of seismic data quality. Although the interval velocity of high-precision seismic inversion in recent years has improved the prediction accuracy of interval velocity to a certain extent, the upper limit and lower limit of rock velocity in the above methods are calculated by empirical formula from the Gulf of Mexico area, thus the calculation results are often with larger error in the areas with abnormal low-velocity lithology or abnormal high-velocity lithology, which leads to the error of pressure prediction results [17]. In this paper, the upper limit and lower limit of rock velocity are obtained by using the equivalent medium theory, which improves the adaptability of the method and the accuracy of pressure prediction [17]. In the same hydrodynamic system, the densities of oil and gas are smaller than that of water, so their pressure gradients are also smaller. Therefore, after using seismic data and well logging data to predict the formation pressure, further calculation of the pressure gradient can detect the properties of the fluids.

\section{Method and Principles}

\subsection{Fillippone Formula}

The algorithm of the Fillippone Formula is simple and independent of the normal compaction curve. It is suitable for pressure calculation in places with large areas. The expression is:

$$
P_{\mathrm{p}}\left(H_{1}\right)=\frac{\mathcal{V}_{\max }-\boldsymbol{V}_{i}}{\mathcal{V}_{\max }-\boldsymbol{V}_{\min }} \int_{0}^{H} 10 \rho_{\mathrm{b}}(h) g \mathrm{~d} h
$$

where: $P_{\mathrm{p}}$-Formation pressure; $V_{\mathrm{i}}$ - Interval velocity of the $i^{\text {th }}$ interval; $\rho_{\mathrm{b}}$-Density of sediment at a certain depth; $V_{\max }$ - The velocity when porosity is approaching zero (rock matrix velocity), which is close to the upper limit of rock velocity; $V_{\min }$-The velocity when rock rigidity approaching zero (fluid velocity), this velocity is close to the lower limit of rock velocity; $H_{1}$-Depth of target layer; $g$-Acceleration of gravity [17].

Hereinto, the upper limit and lower limit of rock velocity are obtained by Fillippone according to the fitting result of stratigraphic statistics of the Gulf of Mexico and other areas. The empirical formula is:

$$
\left\{\begin{array}{l}
v_{\text {max }}=1.4 v_{\mathrm{R} 0}+3 k t \\
\boldsymbol{v}_{\text {min }}=0.7 v_{\mathrm{R} 0}+0.5 k t
\end{array}\right.
$$

where: $t$-Two-way time; $V_{\mathrm{R} 0}$ - Intercept of root mean square velocity varying with $t ; k$-Slope [17].

\subsection{Calculation of Upper Limit and Lower Limit of Rock Velocity Based on Equivalent Medium Theory}

Voigt and Reuss (1929) proposed algorithms for calculating the upper limit and lower limit of the modulus of mineral particle and pore mixture, which are called Voigt Model and Reuss Model respectively [18, 19].

Voigt Model is mainly used to estimate the upper limit of the equivalent modulus of mixed materials, while Reuss Model is mainly utilized to estimate the lower limit [17]. Assuming that the rock matrix is composed of $N$ kinds of materials, the upper limits of bulk modulus and shear modulus are as follows:

$$
\left\{\begin{array}{l}
K_{\mathrm{v}}=\sum_{i=1}^{N} K_{i} V_{i} \\
\mu_{\mathrm{v}}=\sum_{i=1}^{N} \mu_{i} V_{i}
\end{array}\right.
$$

where: $K \mathrm{i}-$ Bulk modulus of the $i^{\text {th }}$ component; $\mu_{\mathrm{i}}$ - Shear modulus of the $i^{\text {th }}$ component; $V_{\mathrm{i}}-$ Volume component of the $i^{\text {th }}$ component [17].

According to the relationship between the propagation velocity of seismic wave and the elastic modulus of material, the upper limit of rock velocity can be determined as follows:

$$
v_{\text {Voigt }}=\sqrt{\frac{K_{\mathrm{v}}+\frac{4}{3} \mu_{\mathrm{v}}}{\rho}}
$$

Reuss (1929) proposed the equivalent modulus model of particle suspension in porous rocks as:

$$
\begin{gathered}
\frac{1}{K_{R}}=\sum_{i=1}^{N} \frac{V_{i}}{K_{i}} \\
\frac{1}{\mu_{R}}=\sum_{i=1}^{N} \frac{V_{i}}{\mu_{i}}
\end{gathered}
$$

where: $K_{R}$ - Low limit of rock bulk modulus; $\mu \mathrm{R}$ - Low limit of rock shear modulus [17-19].

Then, the lower velocity limit of rocks saturated with fluid is:

$$
v_{\text {Reuss }}=\sqrt{\frac{K_{\mathrm{R}}+\frac{4}{3} \mu_{\mathrm{R}}}{\rho}}
$$

In Figure 1, the black curve above is the upper limit of the rock velocity versus porosity change calculated by Voigt Model, and the lower curve is the lower limit calculated by Reuss Model. This means that when the composition and porosity of the rock are fixed, no matter how the combination style of compositions changes, the maximum velocity will not exceed the Voigt line and the minimum velocity will not be less than the Reuss line [17]. 


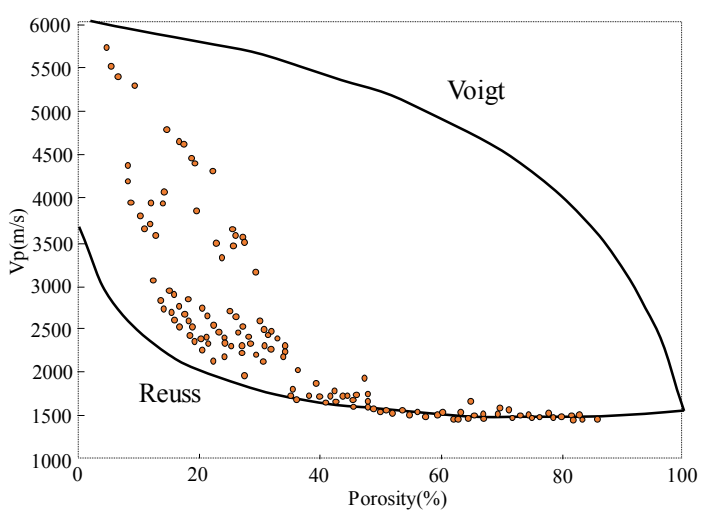

Figure 1. Relationship between upper and lower limits of $P$-wave velocity and porosity.

When Equation (4) and Equation (6) are substituted into Equation (1), the formation pressure of any lithologic formation can be calculated [17].

\subsection{Realization of Formation Pressure Gradient Prediction Method}

Using seismic data to predict pressure gradient requires drilled wells in the study area and logging interpretation results, that is, porosity, content of each lithology, percentage content of pore fluid, etc. The Voigt Model and Reuss Model in the equivalent medium theory can be used to calculate the upper limit curve and lower limit curve of rock velocity at the well point [17]. Afterwards, using the upper limit curve, the lower limit curve and the original acoustic wave curve, the volumes of the upper limit of rock velocity, the lower limit of rock velocity and the conventional impedance can be obtained. Based on these three types of volumes, the formation pressure volume of the whole area can be predicted by Fillipptone Formula. Subsequently, as the formation pressure is a function of depth, the pressure coefficient and pressure gradient can be obtained from the derivation of the depth. The typical pressure gradient of water, oil and gas are $0.01 \mathrm{MPa} / \mathrm{m}, 0.0079 \mathrm{MPa} / \mathrm{m}$ and $0.0018 \mathrm{MPa} / \mathrm{m}$ respectively, the hydrocarbon-bearing property of the reservoir can be judged according to the actual pressure gradient.

\section{Case Analysis}

The study area is located in the Ruokai Basin, Myanmar, Bay of Bengal. Several large biogenic gas fields have been found in the basin, and the target layer is Pliocene deep-sea turbidite sandstone. A large number of slope channel complexes are developed in the study area, including superimposed channel sandstones and synchronous over-shore sediments and natural levees. On seismic section, the channel sandstone has obvious characteristics of undercutting and strong amplitude reflection (Figure 2a). In early researches, based on the frequency information, the effect of hydrocarbon detection method based on frequency spectrum is not obvious. Through further analysis, the amplitude spectrum is extracted from the target layer (Figure $2 b$ ). Since the spectrum difference between gas wells and dry wells is smaller and there is no obvious phenomenon of "low frequency resonance, high frequency attenuation", it is difficult to predict hydrocarbons with spectrum attributes for hydrocarbon detection. Therefore, we try to use seismic data to calculate the pressure gradient, so as to predict hydrocarbons.
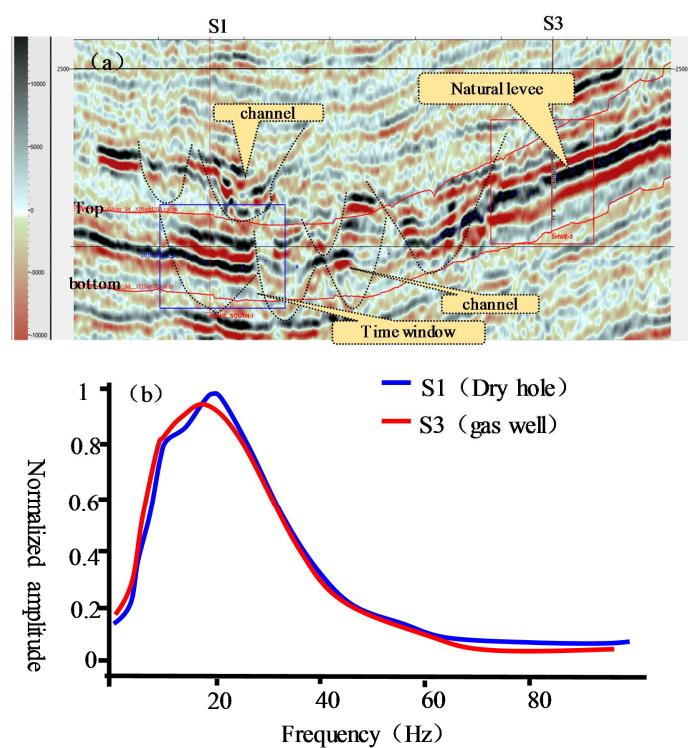

Figure 2. Seismic characteristics and frequency spectrum of sand body.

(a) Seismic reflection characteristics of sand body; (b) Amplitude spectrum characteristics of gas well and dry well

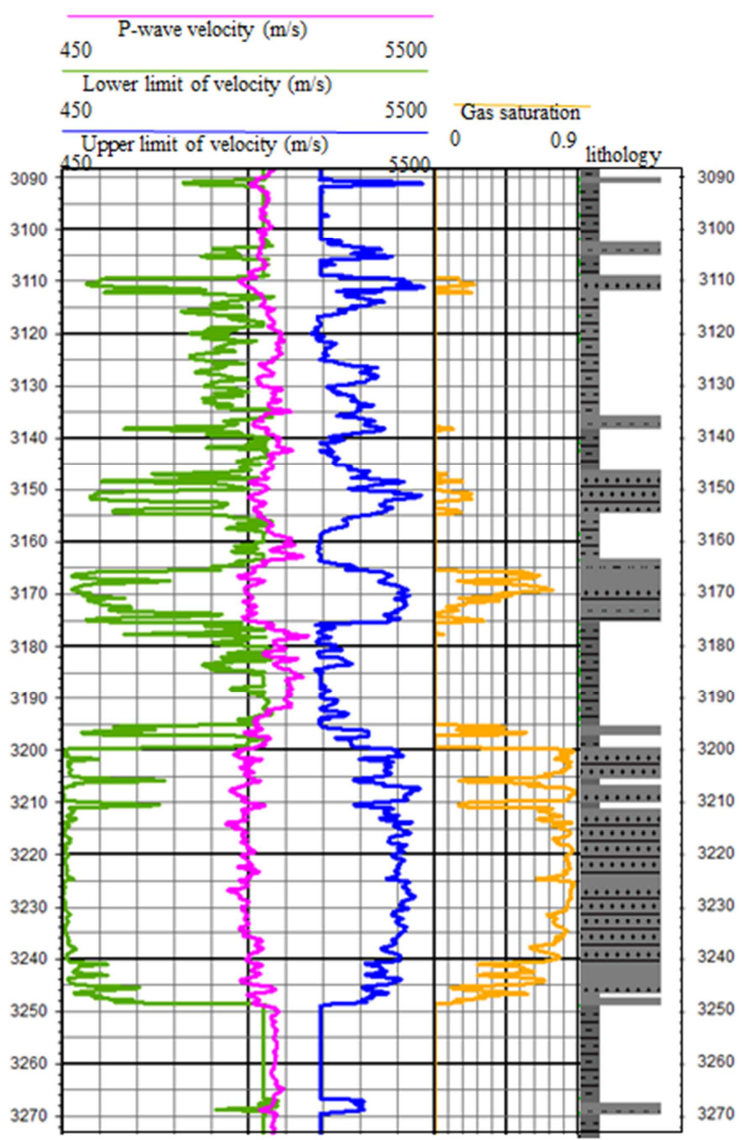

Figure 3. Upper limit and lower limit of rock velocity obtained by Voigt and Reuss models in Well S3. 


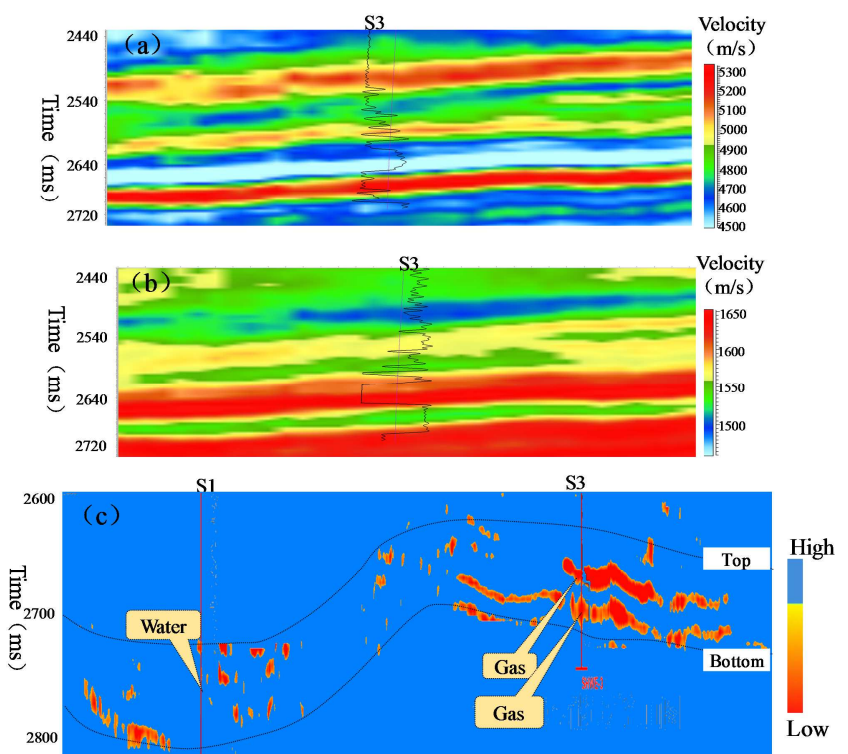

Figure 4. Upper limit profile andlower limit profile of rock velocityand pressure gradient profile.

(a) Upper limit profile of rock velocity; (b) Lower limit profile of rock velocity; (c) Pressure gradient profile

\subsection{Calculation of Upper and Lower Limits of Rock Velocity}

The target layer Pliocene in the study area is mainly interbedding sandstone and mudstone, with well-developed sand bodies. The equivalent medium theory elastic modulus boundary algorithms (from Voigt and Reuss) is used to calculate the upper and lower limit curves of rock velocity in Well S3 (Figure 3), the pink curve is the original velocity curve, and the green curve is the lower limit curve of rock velocity, which reflects the velocity of pure fluid. The blue curve is the upper limit of rock velocity, which reflects the velocity of rock matrix. The yellow curve is the gas saturation from well logging interpretation. At intervals of $3165-3175 \mathrm{~m}$ and $3200-3250 \mathrm{~m}$, the interval velocity is low, while the lower limit of rock velocity is low and the upper limit of rock velocity is high, which shows that the rock matrix of sandstone is characterized by high velocity. As the gas saturation increases (yellow curve), the velocity decreases. The upper limit and lower limit of rock velocity predicted by the equivalent medium theory are extrapolated to the whole area by interpolation or seismic inversion, and the upper limit data volume (Figure 4a) and lower limit data volume (Figure $4 \mathrm{~b}$ ) of rock velocity in the whole region are obtained. Near the target layer of $2640 \mathrm{~ms}$, the upper limit value and lower limit value of rock velocities show obvious mirror characteristics on section. The lower limit data volume of rock velocity is obviously low, while the upper limit data volume of rock velocity is obviously high, which reflects higher gas saturation. By substituting the data volumes of Figure $4 \mathrm{a}$ and Figure $4 \mathrm{~b}$ into Equation (1), the formation pressure profile can be obtained, and the pressure gradient profile can be further obtained by derivation. Figure $4 \mathrm{c}$ shows the pressure gradient profile crossing water well and gas well in the study area. In the target layer of Well S3, there are two obvious areas with low pressure gradient, which are revealed as gas layers. There is no obvious abnormally low value in the target layer of Well S1, which is revealed as water layer by drilling. Therefore, oil and gas can be predicted by pressure gradient.

\subsection{Analysis of Prediction Result}

\subsubsection{Prediction of Drilled Area}

Figure 5 shows the pressure prediction result. In the study area, Well S1 is a water well, and Wells S3, S4 and S6 are gas wells. According to the prediction result, Well S3 is located in an extensive area with obvious low pressure gradient, Well S4 is located in a local low-value area of pressure gradient, Well $\mathrm{S} 1$ is located outside the low value of pressure gradient and has entered into the high value area, and Well S6 is located in an obvious high-value area. As mentioned above, the typical pressure gradient of water, oil and gas are $0.01 \mathrm{MPa} / \mathrm{m}, 0.0079$ $\mathrm{MPa} / \mathrm{m}$ and $0.0018 \mathrm{MPa} / \mathrm{m}$ respectively. According to the predicted values, Wells S3 and S4 are oil wells, Wells S1 and S6 are water wells, and only Well S6 of the three wells is not in accordance with the drilling result. Table 1 shows the comparison between the measured and predicted pressures. The coincidence rate of predicted pressure and the actual drilling results of Wells S1 and S4 are larger than 90\%, which are the highest. The coincidence rate of Well S3 is 72\%. Well S6 is predicted to be a water well, but the coincident rate is only $62 \%$. The average value of the coincidence rate of hydrocarbon detection in this area by using pressure gradient is more than $70 \%$.
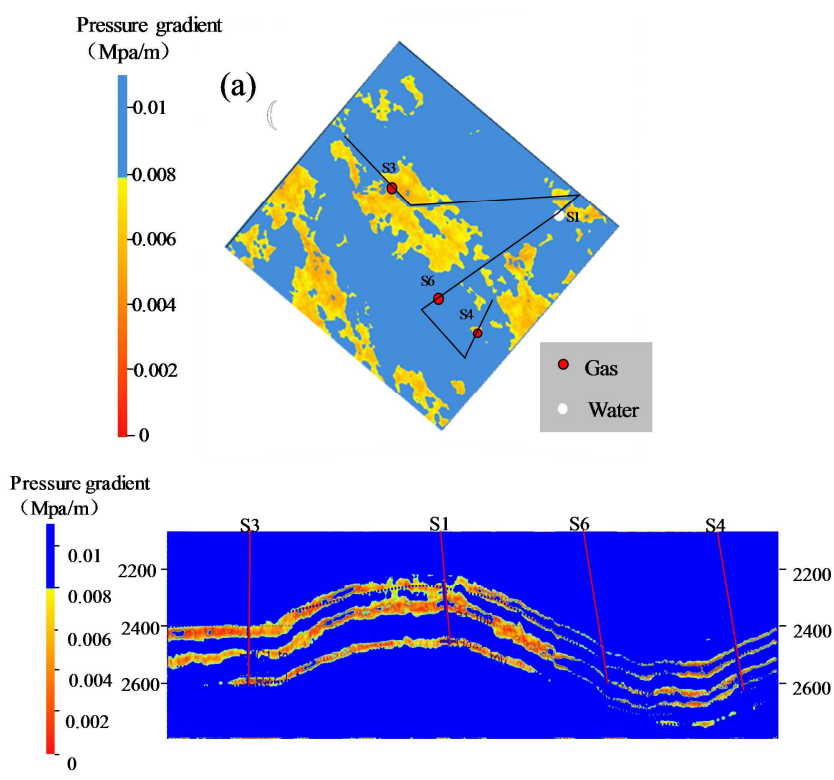

(a) Planar map of pressure gradient; (b) Profile of pressure gradient

Figure 5. Pressure prediction result. 
Table 1. Comparison between the measured and predicted pressure gradients.

\begin{tabular}{lllll}
\hline Well & Reservoir type & Measured pressure gradient $(\mathbf{M P a} / \mathbf{m})$ & Predicted pressure gradient $(\mathbf{M P a} / \mathbf{m})$ & Prediction accuracy \\
\hline S1 & Water layer & 0.01 & 0.0104 & $96 \%$ \\
S3 & Gas layer & 0.00187 & 0.0024 & $72 \%$ \\
S4 & Gas layer & 0.00187 & 0.0077 & $92.5 \%$ \\
S6 & Gas layer & 0.00187 & 0.012 & $62 \%$ \\
\hline
\end{tabular}

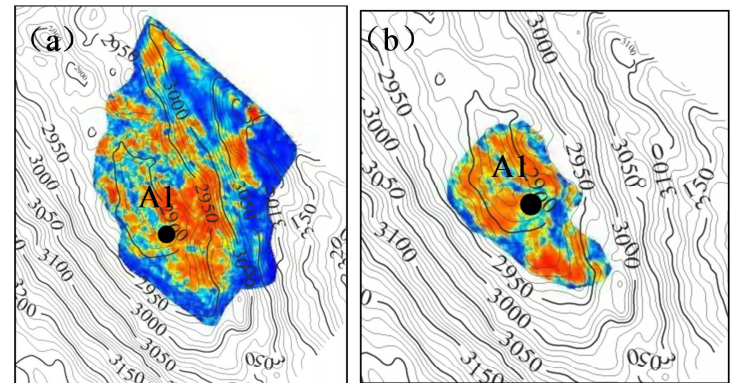

A1

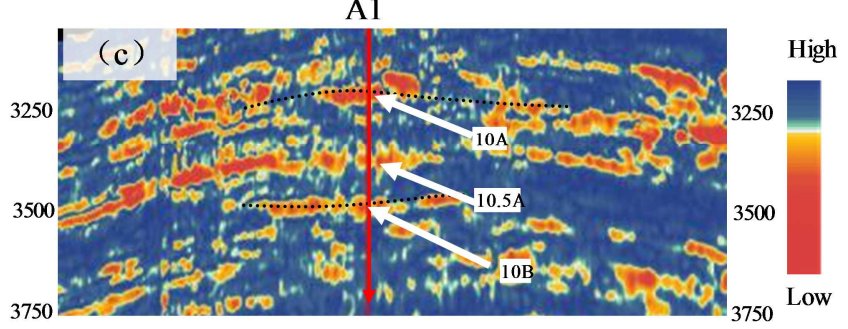

Figure 6. Prediction results in drilled wells.

(a) Superposition of hydrocarbon prediction result and structure of $10 \mathrm{~A}$ sandbody; (b) Superposition of hydrocarbon prediction result and structure of 10B sandbody; (c) Hydrocarbon prediction profile by pressure gradient

\subsubsection{Actual Drilling Results}

The prediction result is extended to the undrilled regions in the adjacent area, and one well is deployed (Figure 6). Three sets of sandbodies are predicted, among which sandbody $10 \mathrm{~A}$ and 10B have more clear low pressure gradient area and are predicted to be better hydrocarbon-bearing areas. Better results have been achieved in drilled wells. Hereinto, there are $26.17 \mathrm{~m} / 13$ gas layers interpreted in the sandbody $10 \mathrm{~A}$, with average porosity of $19.74 \%$ and average water saturation of $36.63 \%$; there are $19.05 \mathrm{~m} / 8$ gas layers interpreted in the sandbody $10 \mathrm{~B}$, with average porosity of $24.15 \%$ and average water saturation of $23.15 \%$.

\section{Conclusions}

1) Fluid Pressure gradient attribute is a new method to predict the hydrocarbon, which is based on Equivalent Medium Theory to calculate the upper limit and lower limit of rock velocity precisely in order to solve Fillippone pressure formula. Comparison with traditional experience-formula method, this method will have more usability.

2) While estimating the upper limit and lower limit of rock velocity based on Equivalent Medium Theory, the petrological curves such as DT, density, total porosity, water saturation, gas saturation, oil saturation and the percentage of lithology will be needed, therefore, it is applicable to the area where some wells have been drilled.

\section{References}

[1] LANG X L, PENG S M, KANG H Q, et al. Application of prestack simultaneous inversion in fluid identification [J]. Geophysical Prospecting for Petroleum, 2010, 49 (2): 164-169.

[2] HUANG R, LIU Z B. Application of prestack simultaneous inversion in sandstone oil reservoir prediction [J]. Progress in Geophysics, 2013, 28 (1): 380-386.

[3] GAO Y, ZHU Y K, ZHAO H, et al. Application of prestack simultaneous inversion technique in effective reservoir prediction of sand-gravel body [J]. Geophysical Prospecting for Petroleum, 2013, 52 (2): 223-228.

[4] XIE Y L. Application study of 3D hydrocarbon detection technology in Fenggu area in western Sichuan basin [J]. Natural Gas Industry, 2006, 26 (3): 46-49.

[5] ZHAO W J, YANG W Y, ZHANG Q F, et al. A frequency AVO method for hydrocarbon detection. [J]. Oil Geophysical Prospecting, 2012, 47 (3): 436-441.

[6] ZHANG Z W, HE Z H, TANG X R. Prediction of reef facies reservoirs based on low-frequency shadow and waveimpedance inversion [J]. Geophysical Prospecting for Petroleum, 2010, 49 (6): 611-615.

[7] LIU W, HE Z H, CHEN X H, et al. Detection of Oil and Gas in Turbidite Fan System by Low-Frequency Shadows Method [J]. Xinjiang Petroleum Geology, 2011 (6): 609-612.

[8] WANG X P, HE Z H, XIONG X J. Namerical simulation of low frequency adjoint-shadows based on wavelet attenuation and hydrocarbon detection [J]. Petroleum Geology and Oilfield Development in Daqing, 2011, 30 (1): 157-160.

[9] GEERTSMA J, SMIT D C. Some aspects of elastic wave propagation in fluid-saturated porous solids [J]. Geophysics, 1961, 26: 169-181.

[10] THEOdoros K, CLIVE M C. Why is the Biot's low compressional wave not observed in real rocks? [J]. Geophysics, 1988, 53: 1605-1609.

[11] LIANG X W, SHI X H, CAI F X, at al. A multi-phase theory based hydrocarbon detection method [J]. Oil Geophysical Prospecting, 2002, 25 (6): 32-35.

[12] ZHANG Y B. Biot theory in application to seismic prospecting [J]. Geophysical Prospecting for Petroleum, 1994, 33 (4): 29-38.

[13] FILLIPPONE W R. Estimation of formation parameters and the prediction of overpressures from seismic data [R]. SEG Technical Program Expanded Abstracts, 1982 (1): 502-503.

[14] RUBEN D, MARTINEZ. Deterministic estimation of porosity and formation pressure from seismic data [R]. SEG Technical Program Expanded Abstracts, 1999: 461-464. 
[15] LIU Z, ZHANG W X, ZHANG H F, at al. An analysis of abnormal formation pressures of Paleogene in the north sag of Liaoxi depression [J]. University of Petroleum, 1993, 14 (1): 14-24.

[16] YUN M H. Formation pressure prediction using seismic data [J]. Oil Geophysical Prospecting, 1996, 31 (4): 5 75-586.

[17] Qian L P, Wang X, Li F, et al. Formation pore pressure prediction using Fillipone formula combined with the equivalent medium theory [J]. Oil Geophysical Prospection, 2018, 53 (Sup. 2): 224-229.

[18] VOIGT W. Ueber die Beziehung Zwischen den beiden Elastizitatsconstanten isotroper Korper [J]. Annalen Der Physi, 1889, 274 (12): 573-587.

[19] REUSS A, A NGEW Z. Berechnung del fliessgrenze von misch- kristallen auf grund der plastizitatbedingung for einkristalle [J]. Math Mech, 1929, 9 (1): 49-58. 